PROCEEDINGS OF THE

AMERICAN MATHEMATICAL SOCIETY

Volume 133, Number 4, Pages 965-974

S 0002-9939(04)07629-4

Article electronically published on November 19, 2004

\title{
ON THE BETTI NUMBERS OF SIGN CONDITIONS
}

\author{
SAUGATA BASU, RICHARD POLLACK, AND MARIE-FRANÇOISE ROY
}

(Communicated by Michael Stillman)

Abstract. Let $\mathrm{R}$ be a real closed field and let $\mathcal{Q}$ and $\mathcal{P}$ be finite subsets of $\mathrm{R}\left[X_{1}, \ldots, X_{k}\right]$ such that the set $\mathcal{P}$ has $s$ elements, the algebraic set $Z$ defined by $\wedge_{Q \in \mathcal{Q}} Q=0$ has dimension $k^{\prime}$ and the elements of $\mathcal{Q}$ and $\mathcal{P}$ have degree at most $d$. For each $0 \leq i \leq k^{\prime}$, we denote the sum of the $i$-th Betti numbers over the realizations of all sign conditions of $\mathcal{P}$ on $Z$ by $b_{i}(\mathcal{P}, \mathcal{Q})$. We prove that

$$
b_{i}(\mathcal{P}, \mathcal{Q}) \leq \sum_{j=0}^{k^{\prime}-i}\left(\begin{array}{l}
s \\
j
\end{array}\right) 4^{j} d(2 d-1)^{k-1}
$$

This generalizes to all the higher Betti numbers the bound $\left(\begin{array}{c}s \\ k^{\prime}\end{array}\right) O(d)^{k}$ on $b_{0}(\mathcal{P}, \mathcal{Q})$. We also prove, using similar methods, that the sum of the Betti numbers of the intersection of $Z$ with a closed semi-algebraic set, defined by a quantifier-free Boolean formula without negations with atoms of the form $P \geq 0$ or $P \leq 0$ for $P \in \mathcal{P}$, is bounded by

$$
\sum_{i=0}^{k^{\prime}} \sum_{j=0}^{k^{\prime}-i}\left(\begin{array}{l}
s \\
j
\end{array}\right) 6^{j} d(2 d-1)^{k-1}
$$

making the bound $s^{k^{\prime}} O(d)^{k}$ more precise.

\section{INTRODUCTION}

Let $\mathrm{R}$ be a real closed field. For an element $a \in \mathrm{R}$ we define

$$
\operatorname{sign}(a)= \begin{cases}0 & \text { if } a=0 \\ 1 & \text { if } a>0 \\ -1 & \text { if } a<0\end{cases}
$$

Let $\mathcal{Q}$ and $\mathcal{P}$ be finite subsets of $\mathrm{R}\left[X_{1}, \ldots, X_{k}\right]$. A sign condition on $\mathcal{P}$ is an element of $\{0,1,-1\}^{\mathcal{P}}$.

For $r>0$ we define the sets $Z$ and $Z_{r}$ by

$$
Z=\mathcal{R}\left(\bigwedge_{Q \in \mathcal{Q}} Q=0\right)=\left\{x \in \mathrm{R}^{k} \mid \bigwedge_{Q \in \mathcal{Q}} Q(x)=0\right\}, Z_{r}=Z \cap B(0, r) .
$$

Received by the editors July 3, 2002 and, in revised form, October 10, 2003.

2000 Mathematics Subject Classification. Primary 14P10; Secondary 14P25.

Key words and phrases. Betti numbers, sign conditions, semi-algebraic sets.

The first author was supported in part by NSF grant CCR-0049070 and an NSF Career Award 0133597.

The second author was supported in part by NSA grant MDA904-01-1-0057 and NSF grants CCR-9732101 and CCR-0098246. 
The realization of the sign condition $\sigma$ over $Z, \mathcal{R}(\sigma, Z)$, is the basic semi-algebraic set

$$
\left\{x \in \mathrm{R}^{k} \mid \bigwedge_{Q \in \mathcal{Q}} Q(x)=0 \wedge \bigwedge_{P \in \mathcal{P}} \operatorname{sign}(P(x))=\sigma(P)\right\} .
$$

The realization of the sign condition $\sigma$ over $Z_{r}, \mathcal{R}\left(\sigma, Z_{r}\right)$, is the basic semi-algebraic set $\mathcal{R}(\sigma, Z) \cap B(0, r)$.

For the rest of the paper, we fix an open ball $B(0, r)$ with center 0 and radius $r$ big enough so that, for every sign condition $\sigma, \mathcal{R}(\sigma, Z)$ and $\mathcal{R}\left(\sigma, Z_{r}\right)$ are homeomorphic. This is always possible by the local conical structure at infinity of semi-algebraic sets ([5], page 225).

A closed and bounded semi-algebraic set $S \subset \mathrm{R}^{k}$ is semi-algebraically triangulable (see [5]), and we denote by $H_{i}(S)$ the $i$-th simplicial homology group of $S$ with rational coefficients. The groups $H_{i}(S)$ are invariant under semi-algebraic homeomorphisms and coincide with the corresponding singular homology groups when $\mathrm{R}=\mathbb{R}$. We denote by $b_{i}(S)$ the $i$-th Betti number of $S$ (that is, the dimension of $H_{i}(S)$ as a vector space), and by $b(S)$ the sum $\sum_{i} b_{i}(S)$. For a closed but not necessarily bounded semi-algebraic set $S \subset \mathrm{R}^{k}$, we will denote by $H_{i}(S)$ the $i$-th simplicial homology group of $S \cap \overline{B(0, r)}$, where $r$ is sufficiently large. This is welldefined using the local conical structure at infinity of semi-algebraic sets ([5], page $225)$.

The definition of homology groups of arbitrary semi-algebraic sets in $\mathrm{R}^{k}$ requires some care, and several possibilities exist. In this paper, we define the homology groups of realizations of sign conditions as follows. Let $\sigma \in\{0,1,-1\}^{\mathcal{P}}$, and let $S_{t} \subset$ $\mathrm{R}^{k}, t \in(0, \infty]$ be any semi-algebraic family of closed and bounded sets, satisfying $\bigcup_{0<t} S_{t}=\mathcal{R}\left(\sigma, Z_{r}\right)$ and $t_{1}>t_{2} \Rightarrow S_{t_{1}} \subset S_{t_{2}}$. It follows from Hardt's triviality theorem [6] that there exists $t_{0}>0$ such that for all $t \in\left(0, t_{0}\right], S_{t}$ is homeomorphic to $S_{t_{0}}$. We define $H_{i}(\mathcal{R}(\sigma, Z))$ to be the simplicial homology group $H_{i}\left(S_{t_{0}}\right)$ with coefficients in $\mathbb{Q}$. It is easy to see (again using Hardt's triviality theorem) that $H_{i}(\mathcal{R}(\sigma, Z))$ does not depend on the choice of the semi-algebraic family $S_{t}$ and also that it is invariant under semi-algebraic homeomorphisms. Finally, in the case that $\mathrm{R}=\mathbb{R}, H_{i}(\mathcal{R}(\sigma, Z))$ is isomorphic to the $i$-th singular homology group of $\mathcal{R}(\sigma, Z)$ using the fact that the singular homology of a subset of $\mathbb{R}^{k}$ is isomorphic to the direct limit of the singular homology groups of its compact subsets [9].

Let $b_{i}(\sigma)$ denote the $i$-th Betti number of $\mathcal{R}(\sigma, Z)$, i.e., the dimension of $H_{i}(\mathcal{R}(\sigma, Z))$ as a $\mathbb{Q}$ vector space, and let $b_{i}(\mathcal{Q}, \mathcal{P})=\sum_{\sigma} b_{i}(\sigma)$. Note that $b_{0}(\mathcal{Q}, \mathcal{P})$ is the total number of semi-algebraically connected components of the realizations of all realizable sign conditions of $\mathcal{P}$ over $Z$.

We write $b_{i}\left(d, k, k^{\prime}, s\right)$ for the maximum of $b_{i}(\mathcal{Q}, \mathcal{P})$ over all $\mathcal{Q}, \mathcal{P}$ where $\mathcal{Q}$ and $\mathcal{P}$ are finite subsets of $\mathrm{R}\left[X_{1}, \ldots, X_{k}\right]$, whose elements have degree at most $d, \#(\mathcal{P})=s$ (i.e. $\mathcal{P}$ has $s$ elements) and the algebraic set $Z$ has real dimension $k^{\prime}$.

In [3], it was shown that, $b_{0}\left(d, k, k^{\prime}, s\right)=\left(\begin{array}{c}s \\ k^{\prime}\end{array}\right) O(d)^{k}$. The main point in this paper is to prove an extension of this result by obtaining bounds for $b_{i}\left(d, k, k^{\prime}, s\right)$, for each $i, 0 \leq i \leq k^{\prime}$. Namely, we prove:

\section{Theorem 1.1.}

$$
b_{i}\left(d, k, k^{\prime}, s\right) \leq \sum_{j=0}^{k^{\prime}-i}\left(\begin{array}{l}
s \\
j
\end{array}\right) 4^{j} d(2 d-1)^{k-1} .
$$


The bound in [3] is proved by using a general position argument. The given polynomials are perturbed using infinitesimals so as to put them in general position - i.e. so that no more than $k^{\prime}$ of the polynomials in $\mathcal{P}$ have a common real zero in $Z$. The main ideas behind the proofs of the results in this paper are very different. We use an inductive argument based on the Mayer-Vietoris sequence. The starting point of the induction is a dimension argument: namely, we use the fact that the $i$-th Betti number of a semi-algebraic set is zero when $i$ is greater than its dimension. Notice that for $i=0$, Theorem 1.1 gives a more precise bound than the one in [3]. In [1] separate bounds on the individual Betti numbers of basic closed semi-algebraic sets were proved using a spectral sequence argument. The spectral sequences described there suggest the inequalities proved in Proposition 2 below, but they hide the direct induction that we are performing here.

We start with preliminaries, prove Theorem 1.1 in Section 3, and in Section 4 study the sum of Betti numbers of closed semi-algebraic sets.

\section{Preliminaries}

We use two main ingredients: the Oleinik-Petrovski/Thom/Milnor bound on the sum of the Betti numbers of algebraic sets and the Mayer-Vietoris long exact sequence. Additionally, we will use certain tools from real algebraic geometry.

Let $b(k, d)$ be the maximum of the sum of the Betti numbers of any algebraic set defined by polynomials of degree $d$ in $\mathrm{R}^{k}$. The Oleinik-Petrovski/Thom/Milnor [7, 10, 8] bound is the following:

$$
b(k, d) \leq d(2 d-1)^{k-1} .
$$

We use extensively the inequalities in the following Proposition 1, which are easy consequences of the exactness of the Mayer-Vietoris sequence of homology groups [9] : if $S_{1}, S_{2}$ are two closed and bounded semi-algebraic sets, then there exists the following long exact sequence of homology groups:

$$
\cdots \rightarrow H_{i}\left(S_{1} \cap S_{2}\right) \rightarrow H_{i}\left(S_{1}\right) \oplus H_{i}\left(S_{2}\right) \rightarrow H_{i}\left(S_{1} \cup S_{2}\right) \rightarrow H_{i-1}\left(S_{1} \cap S_{2}\right) \rightarrow \cdots .
$$

Proposition 1. Let $S_{1}, S_{2}$ be two closed and bounded semi-algebraic sets. Then,

$$
\begin{gathered}
b_{i}\left(S_{1}\right)+b_{i}\left(S_{2}\right) \leq b_{i}\left(S_{1} \cup S_{2}\right)+b_{i}\left(S_{1} \cap S_{2}\right), \\
b_{i}\left(S_{1} \cup S_{2}\right) \leq b_{i}\left(S_{1}\right)+b_{i}\left(S_{2}\right)+b_{i-1}\left(S_{1} \cap S_{2}\right), \\
b_{i}\left(S_{1} \cap S_{2}\right) \leq b_{i}\left(S_{1}\right)+b_{i}\left(S_{2}\right)+b_{i+1}\left(S_{1} \cup S_{2}\right) .
\end{gathered}
$$

We perturb polynomials by various infinitesimals so that our geometric objects live over the field of algebraic Puiseux series in these infinitesimals. We denote by $\mathrm{R}\langle\zeta\rangle$ the real closed field of algebraic Puiseux series in $\zeta$ with coefficients in $\mathrm{R}$ [4. The sign of a Puiseux series in $\mathrm{R}\langle\zeta\rangle$ agrees with the sign of the coefficient of the lowest degree term in $\zeta$. This order makes $\zeta$ infinitesimal: $\zeta$ is positive and smaller than any positive element of $\mathrm{R}$. When $a \in \mathrm{R}\langle\zeta\rangle$ is bounded by an element of $\mathrm{R}$, $\lim _{\zeta}(a)$ is the constant term of $a$, obtained by substituting 0 for $\zeta$ in $a$.

Let $\mathrm{R}$ denote a real closed field and $\mathrm{R}^{\prime}$ a real closed field containing $\mathrm{R}$. Given a semi-algebraic set $S$ in $\mathrm{R}^{k}$, the extension of $S$ to $\mathrm{R}^{\prime}$, denoted $\operatorname{Ext}\left(S, \mathrm{R}^{\prime}\right)$, is the

semi-algebraic subset of $\mathrm{R}^{\prime k}$ defined by the same quantifier free formula that defines $S$. The set $\operatorname{Ext}\left(S, \mathrm{R}^{\prime}\right)$ is well defined (i.e. it only depends on the set $S$ and not on the quantifier free formula chosen to describe it). This is an easy consequence 
of the transfer principle [5]. Moreover, the Betti numbers are not changed after extension: $b_{i}(S)=b_{i}\left(\operatorname{Ext}\left(S, \mathrm{R}^{\prime}\right)\right)$ (see [4], Chapter 6).

\section{Bounds on Betti Numbers of BAsiC SEMi-AlgEBRAIC SETS: Proof OF THEOREM 1.1}

Let $S_{1}, \ldots, S_{s} \subset \mathrm{R}^{k}$ be closed semi-algebraic sets, contained in a closed bounded semi-algebraic set $T$ of dimension $k^{\prime}$. For $1 \leq t \leq s$, we let

$$
S_{\leq t}=\bigcap_{1 \leq j \leq t} S_{j}, \quad S^{\leq t}=\bigcup_{1 \leq j \leq t} S_{j} .
$$

Also, for $J \subset\{1, \ldots, s\}, J \neq \emptyset$, let

$$
S_{J}=\bigcap_{j \in J} S_{j}, S^{J}=\bigcup_{j \in J} S_{j}
$$

Finally, let $S^{\emptyset}=T$.

The following proposition, Proposition 2 plays a key role in the proofs of our theorems. The first part of the proposition bounds the Betti numbers of a union of $s$ semi-algebraic sets in $R^{k}$ in terms of the Betti numbers of the intersections of the sets taken at most $k$ at a time. In some simple situations the Betti numbers of a union of $s$ sets are easy to bound. For instance, when the sets are such that all non-empty intersections amongst them are contractible, a classical result of topology, the nerve lemma, gives us a bound on the individual Betti numbers of the union. The nerve lemma states that the homology groups of such a union are isomorphic to the homology groups of a combinatorially defined simplicial complex, the nerve complex. The nerve complex has $s$ vertices, and thus the $i$-th Betti number is bounded by $\left(\begin{array}{c}s \\ i+1\end{array}\right)$. The first part of the proposition can be thought of as a generalization of this bound to the case when the intersections are not topologically trivial. The second part of the proposition is a dual version of the first, with unions being replaced by intersections and vice-versa, with an additional complication arising from the fact that the empty intersection, corresponding to the base case of the induction, is an arbitrary real algebraic variety of dimension $k^{\prime}$, which is generally not contractible.

Proposition 2. For $0 \leq i \leq k^{\prime}$,

$$
\begin{gathered}
b_{i}\left(S^{\leq s}\right) \leq \sum_{j=1}^{i+1} \sum_{J \subset\{1, \ldots s\}, \#(J)=j} b_{i-j+1}\left(S_{J}\right), \\
b_{i}\left(S_{\leq s}\right) \leq b_{k^{\prime}}\left(S^{\emptyset}\right)+\sum_{j=1}^{k^{\prime}-i} \sum_{J \subset\{1, \ldots, s\}, \#(J)=j}\left(b_{i+j-1}\left(S^{J}\right)+b_{k^{\prime}}\left(S^{\emptyset}\right)\right) .
\end{gathered}
$$

Proof of inequality (3.1). We prove the claim by induction on $s$. The statement is clearly true for $s=1$.

Using Proposition 1(2.3), we have that

$$
b_{i}\left(S^{\leq s}\right) \leq b_{i}\left(S^{\leq s-1}\right)+b_{i}\left(S_{s}\right)+b_{i-1}\left(S^{\leq s-1} \cap S_{s}\right) .
$$


Applying the induction hypothesis to the set $S^{\leq s-1}$, we deduce that

$$
b_{i}\left(S^{\leq s-1}\right) \leq \sum_{j=1}^{i+1} \sum_{J \subset\{1, \ldots, s-1\}, \#(J)=j} b_{i-j+1}\left(S_{J}\right) .
$$

Next, we apply the induction hypothesis to the set

$$
S^{\leq s-1} \cap S_{s}=\cup_{1 \leq j \leq s-1}\left(S_{j} \cap S_{s}\right)
$$

and get that

$$
b_{i-1}\left(S^{\leq s-1} \cap S_{s}\right) \leq \sum_{j=1}^{i} \sum_{J \subset\{1, \ldots, s-1\}, \#(J)=j} b_{i-j}\left(S_{J \cup\{s\}}\right) .
$$

Adding the inequalities obtained above we get

$$
b_{i}\left(S^{\leq s-1}\right)+b_{i}\left(S_{s}\right)+b_{i-1}\left(S^{\leq s-1} \cap S_{s}\right) \leq \sum_{j=1}^{i+1} \sum_{J \subset\{1, \ldots, s\}, \#(J)=j} b_{i-j+1}\left(S_{J}\right) .
$$

Proof of inequality (3.2). We first prove the claim when $s=1$. If $0 \leq i \leq k^{\prime}-1$, the claim is

$$
b_{i}\left(S_{1}\right) \leq b_{k^{\prime}}\left(S^{\emptyset}\right)+\left(b_{i}\left(S_{1}\right)+b_{k^{\prime}}\left(S^{\emptyset}\right)\right) .
$$

If $i=k^{\prime}$, the claim is $b_{k^{\prime}}\left(S_{1}\right) \leq b_{k^{\prime}}\left(S^{\emptyset}\right)$. If the dimension of $S_{1}$ is $k^{\prime}$, consider the closure $V$ of the complement of $S_{1}$ in $T$. The intersection $W$ of $V$ with $S_{1}$, which is the boundary of $S_{1}$, has dimension strictly smaller than $k^{\prime}[5$ (page 53); thus $b_{k^{\prime}}(W)=0$. Using Proposition 1 (2.2), $b_{k^{\prime}}\left(S_{1}\right)+b_{k^{\prime}}(V) \leq b_{k^{\prime}}\left(S^{\emptyset}\right)+b_{k^{\prime}}(W)$, and the claim follows. On the other hand, if the dimension of $S_{1}$ is strictly smaller than $k^{\prime}, b_{k^{\prime}}\left(S_{1}\right)=0$.

The claim is now proved by induction on $s$. Assume that the induction hypothesis (3.2) holds for $s-1$ and for all $0 \leq i \leq k^{\prime}$. From Proposition 12.4) we have

$$
b_{i}\left(S_{\leq s}\right) \leq b_{i}\left(S_{\leq s-1}\right)+b_{i}\left(S_{s}\right)+b_{i+1}\left(S_{\leq s-1} \cup S_{s}\right) .
$$

Applying the induction hypothesis to the set $S_{\leq s-1}$, we deduce that

$$
b_{i}\left(S_{\leq s-1}\right) \leq b_{k^{\prime}}\left(S^{\emptyset}\right)+\sum_{j=1}^{k^{\prime}-i} \sum_{J \subset\{1, \ldots, s-1\}, \#(J)=j}\left(b_{i+j-1}\left(S^{J}\right)+b_{k^{\prime}}\left(S^{\emptyset}\right)\right) .
$$

Next, applying the induction hypothesis to the set, $S_{\leq s-1} \cup S_{s}=\bigcap_{1 \leq j \leq s-1}\left(S_{j} \cup\right.$ $S_{s}$ ), we get that

$$
b_{i+1}\left(S_{\leq s-1} \cup S_{s}\right) \leq b_{k^{\prime}}\left(S^{\emptyset}\right)+\sum_{j=1}^{k^{\prime}-i-1} \sum_{J \subset\{1, \ldots, s-1\}, \#(J)=j}\left(b_{i+j}\left(S^{J \cup\{s\}}\right)+b_{k^{\prime}}\left(S^{\emptyset}\right)\right) .
$$

Adding the inequalities obtained above we get

$$
b_{i}\left(S_{\leq s}\right) \leq b_{k^{\prime}}\left(S^{\emptyset}\right)+\sum_{j=1}^{k^{\prime}-i} \sum_{J \subset\{1, \ldots, s\}, \#(J)=j}\left(b_{i+j-1}\left(S^{J}\right)+b_{k^{\prime}}\left(S^{\emptyset}\right)\right) .
$$


Let $\mathcal{P}=\left\{P_{1}, \ldots, P_{s}\right\}$, and let $\delta$ be a new variable. We consider the field, $\mathrm{R}\langle\delta\rangle$, of algebraic Puiseux series in $\delta$, in which $\delta$ will be an infinitesimal. Let $W_{0}$ (resp. $\left.W_{1}\right)$ be the union of the sets $\mathcal{R}\left(P_{i}^{2}\left(P_{i}^{2}-\delta^{2}\right)=0, \operatorname{Ext}\left(Z_{r}, \mathrm{R}\langle\delta\rangle\right)\right.$ ) (resp. $\mathcal{R}\left(P_{i}^{2}\left(P_{i}^{2}-\delta^{2} \geq 0\right), \operatorname{Ext}\left(Z_{r}, \mathrm{R}\langle\delta\rangle\right)\right)$ with $1 \leq i \leq j$.

\section{Lemma 3.1.}

$$
b_{i}\left(W_{0}\right) \leq\left(4^{j}-1\right) d(2 d-1)^{k-1} .
$$

Proof. Each of the sets $\mathcal{R}\left(P_{i}^{2}\left(P_{i}^{2}-\delta^{2}\right)=0, \operatorname{Ext}\left(Z_{r}, \mathrm{R}\langle\delta\rangle\right)\right)$ is the disjoint union of three algebraic sets, namely

$$
\begin{aligned}
& \mathcal{R}\left(P_{i}=0, \operatorname{Ext}\left(Z_{r}, \mathrm{R}\langle\delta\rangle\right)\right), \\
& \mathcal{R}\left(P_{i}=\delta, \operatorname{Ext}\left(Z_{r}, \mathrm{R}\langle\delta\rangle\right)\right),
\end{aligned}
$$

and

$$
\mathcal{R}\left(P_{i}=-\delta, \operatorname{Ext}\left(Z_{r}, \mathrm{R}\langle\delta\rangle\right)\right) .
$$

Moreover, each Betti number of their union is bounded by the sum of the Betti numbers of all possible non-empty sets that can be obtained by taking, for $1 \leq \ell \leq j$, $\ell$-ary intersections of these algebraic sets using inequality 3.1 of Proposition 2] The number of possible $\ell$-ary intersections is $\left(\begin{array}{l}j \\ \ell\end{array}\right)$. Each such intersection is a disjoint union of $3^{\ell}$ algebraic sets. The sum of the Betti numbers of each of these algebraic sets is bounded by $d(2 d-1)^{k-1}$ by the Oleinik-Petrovski/Thom/Milnor bound (2.1).

Thus, $b_{i}\left(W_{0}\right) \leq \sum_{\ell=1}^{j}\left(\begin{array}{l}j \\ \ell\end{array}\right) 3^{\ell} d(2 d-1)^{k-1}=\left(4^{j}-1\right) d(2 d-1)^{k-1}$.

\section{Lemma 3.2.}

$$
b_{i}\left(W_{1}\right) \leq\left(4^{j}-1\right) d(2 d-1)^{k-1}+b_{i}\left(Z_{r}\right) .
$$

Proof. Let $Q_{i}=P_{i}^{2}\left(P_{i}^{2}-\delta^{2}\right)$ and

$$
F=\mathcal{R}\left(\bigwedge_{1 \leq i \leq j}\left(Q_{i} \leq 0 \vee \bigvee_{1 \leq i \leq j} Q_{i}=0, \operatorname{Ext}\left(Z_{r}, \mathrm{R}\langle\delta\rangle\right)\right) .\right.
$$

Now apply inequality (2.2), noting that $W_{1} \cup F=\operatorname{Ext}\left(Z_{r}, \mathrm{R}\langle\delta\rangle\right), W_{1} \cap F=W_{0}$, since $b_{i}\left(Z_{r}\right)=b_{i}\left(\operatorname{Ext}\left(Z_{r}, \mathrm{R}\langle\delta\rangle\right)\right)$. We get that $b_{i}\left(W_{1}\right) \leq b_{i}\left(W_{1} \cap F\right)+b_{i}\left(W_{1} \cup F\right)=$ $b_{i}\left(W_{0}\right)+b_{i}\left(Z_{r}\right)$. We conclude using Lemma 3.1.

Let $S_{i}=\mathcal{R}\left(P_{i}^{2}\left(P_{i}^{2}-\delta^{2}\right) \geq 0, \operatorname{Ext}\left(Z_{r}, \mathrm{R}\langle\delta\rangle\right)\right), 1 \leq i \leq \ell$, and $S$ be the intersection of the $S_{i}$. Then

\section{Lemma 3.3.}

$$
b_{i}(\mathcal{P}, \mathcal{Q})=b_{i}(S)
$$

Proof. Consider a sign condition $\sigma$ on $\mathcal{P}$ such that, without loss of generality,

$$
\begin{array}{ll}
\sigma\left(P_{i}\right)=0 & \text { if } i=1, \ldots, j, \\
\sigma\left(P_{i}\right)=1 & \text { if } i=j+1, \ldots, \ell, \\
\sigma\left(P_{i}\right)=-1 & \text { if } i=\ell+1, \ldots, s,
\end{array}
$$

and denote by $\overline{\mathcal{R}}(\sigma)$ the subset of $\operatorname{Ext}\left(Z_{r}, \mathrm{R}\langle\delta\rangle\right)$ defined by

$$
\bigwedge_{i=1, \ldots, j} P_{i}(x)=0 \wedge \bigwedge_{i=j+1, \ldots, \ell} P_{i}(x) \geq \delta \wedge \bigwedge_{\ell+1, \ldots, s} P_{i}(x) \leq-\delta .
$$


It follows from our definition of $b_{i}(\sigma)$ and Hardt's triviality theorem [5] that $b_{i}(\sigma)=b_{i}(\overline{\mathcal{R}}(\sigma))$. Note that $S$ is the disjoint union of the $\overline{\mathcal{R}}(\sigma)$ (for the $\sigma$ realizable sign condition) so that $\sum_{\sigma} b_{i}(\sigma)=b_{i}(S)$. On the other hand, by definition, $\sum_{\sigma} b_{i}(\sigma)=b_{i}(\mathcal{P}, \mathcal{Q})$

We are now able to prove Theorem 1.1

Proof of Theorem 1.1. Using inequality 3.2 of Proposition 2, Lemma3.2, and (2.1) which implies, for all $i<k^{\prime}, b_{i}\left(Z_{r}\right)+b_{k^{\prime}}\left(Z_{r}\right) \leq d(2 d-1)^{k-1}$, we deduce that

$$
b_{i}(S) \leq b_{k^{\prime}}\left(Z_{r}\right)+\sum_{j=1}^{k^{\prime}-i}\left(\begin{array}{l}
s \\
j
\end{array}\right)\left(4^{j} d(2 d-1)^{k-1}\right) .
$$

Thus, we have $b_{i}(S) \leq \sum_{j=0}^{k^{\prime}-i}\left(\begin{array}{l}s \\ j\end{array}\right) 4^{j} d(2 d-1)^{k-1}$.

It now follows, using Lemma 3.3, that

$$
b_{i}(\mathcal{P}, \mathcal{Q}) \leq \sum_{j=0}^{k^{\prime}-i}\left(\begin{array}{l}
s \\
j
\end{array}\right) 4^{j} d(2 d-1)^{k-1},
$$

and finally

$$
b_{i}\left(d, k, k^{\prime}, s\right) \leq \sum_{j=0}^{k^{\prime}-i}\left(\begin{array}{l}
s \\
j
\end{array}\right) 4^{j} d(2 d-1)^{k-1} .
$$

\section{Sum of Betti numbers of Closed Semi-Algebraic Sets}

A $(\mathcal{Q}, \mathcal{P})$-closed formula is a formula defined as follows:

- For each $P \in \mathcal{P}, \bigwedge_{Q \in \mathcal{Q}} Q=0 \wedge P=0, \bigwedge_{Q \in \mathcal{Q}} Q=0 \wedge P \geq 0, \bigwedge_{Q \in \mathcal{Q}} Q=$ $0 \wedge P \leq 0$ are $(\mathcal{Q}, \mathcal{P})$-closed formulas.

- If $\Phi_{1}$ and $\Phi_{2}$ are $(\mathcal{Q}, \mathcal{P})$-closed formulas, $\Phi_{1} \wedge \Phi_{2}$ and $\Phi_{1} \vee \Phi_{2}$ are $(\mathcal{Q}, \mathcal{P})$ closed formulas.

Clearly, $\mathcal{R}(\Phi)$, the intersection of the realization of a $(\mathcal{Q}, \mathcal{P})$-closed formula $\Phi$ with $B(0, r)$ is a closed semi-algebraic set. We denote by $b(\Phi)$ the sum of its Betti numbers.

We write $\bar{b}\left(d, k, k^{\prime}, s\right)$ for the maximum of $b(\Phi)$, where $\Phi$ is a $(\mathcal{Q}, \mathcal{P})$-closed formula, $\mathcal{Q}$ and $\mathcal{P}$ are finite subsets of $\mathrm{R}\left[X_{1}, \ldots, X_{k}\right]$, whose elements have degree at most $d, \#(\mathcal{P})=s$ and the algebraic set $\mathcal{R}\left(\bigwedge_{Q \in \mathcal{Q}} Q=0\right)$ has dimension $k^{\prime}$.

In [2], it was shown that $\bar{b}\left(d, k, k^{\prime}, s\right)$ is bounded by $s^{k^{\prime}} O(d)^{k}$. In this section, we prove a more precise bound:

Theorem 4.1.

$$
\bar{b}\left(d, k, k^{\prime}, s\right) \leq \sum_{i=0}^{k^{\prime}} \sum_{j=0}^{k^{\prime}-i}\left(\begin{array}{l}
s \\
j
\end{array}\right) 6^{j} d(2 d-1)^{k-1} .
$$


For the proof of Theorem 4.1, we are going to introduce several infinitesimals. Given an ordered list of polynomials $\mathcal{P}=\left\{P_{1}, \ldots, P_{s}\right\}$ with coefficients in $R$, we introduce $s$ new variables $\delta_{1}, \cdots, \delta_{s}$, and inductively define: $\mathrm{R}\left\langle\delta_{1}, \ldots, \delta_{i+1}\right\rangle=$ $\mathrm{R}\left\langle\delta_{1}, \ldots, \delta_{i}\right\rangle\left\langle\delta_{i+1}\right\rangle$. Note that $\delta_{i+1}$ is infinitesimal with respect to $\delta_{i}$, which is denoted by

$$
\delta_{1} \gg \ldots \gg \delta_{s} .
$$

We define $\mathcal{P}_{>i}=\left\{P_{i+1}, \ldots, P_{s}\right\}$ and

$$
\begin{gathered}
\Sigma_{i}=\left\{P_{i}=0, P_{i}=\delta_{i}, P_{i}=-\delta_{i}, P_{i} \geq 2 \delta_{i}, P_{i} \leq-2 \delta_{i}\right\}, \\
\Sigma_{\leq i}=\left\{\Psi \mid \Psi=\bigwedge_{j=1, \ldots, i} \Psi_{i}, \Psi_{i} \in \Sigma_{i}\right\} .
\end{gathered}
$$

If $\Phi$ is a $(\mathcal{Q}, \mathcal{P})$-closed formula, we denote by $\mathcal{R}_{i}(\Phi)$ the extension of $\mathcal{R}(\Phi)$ to $\mathrm{R}\left\langle\delta_{1}, \ldots, \delta_{i}\right\rangle^{k}$. For $\Psi \in \Sigma_{\leq i}$, we denote by $\mathcal{R}_{i}(\Phi \wedge \Psi)$ the intersection of the realization of $\Psi$ with $\mathcal{R}_{i}(\Phi)$ and by $b(\Phi \wedge \Psi)$ the sum of the Betti numbers of $\mathcal{R}_{i}(\Phi \wedge \Psi)$.

Proposition 3. For every $(\mathcal{Q}, \mathcal{P})$-closed formula $\Phi$,

$$
b(\Phi) \leq \sum_{\Psi \in \Sigma_{\leq s}, \mathcal{R}_{s}(\Psi) \subset \mathcal{R}_{s}(\Phi)} b(\Psi) .
$$

The main ingredient of the proof of Proposition 3 is the following lemma.

Lemma 4.2. For every $(\mathcal{Q}, \mathcal{P})$-closed formula $\Phi$, and every $\Psi \in \Sigma_{\leq i}, b(\Phi \wedge \Psi) \leq$ $\sum_{\psi \in \Sigma_{i+1}} b(\Phi \wedge \Psi \wedge \psi)$.

Proof. Consider the formulas

$$
\begin{aligned}
& \Phi_{1}=\Phi \wedge \Psi \wedge\left(P_{i+1}^{2}-\delta_{i+1}^{2}\right) \geq 0, \\
& \Phi_{2}=\Phi \wedge \Psi \wedge\left(0 \leq P_{i+1}^{2} \leq \delta_{i+1}^{2}\right) .
\end{aligned}
$$

Clearly, $\mathcal{R}_{i+1}(\Phi \wedge \Psi)=\mathcal{R}_{i+1}\left(\Phi_{1} \vee \Phi_{2}\right)$. Using Proposition 11 we have that $b(\Phi \wedge \Psi) \leq b\left(\Phi_{1}\right)+b\left(\Phi_{2}\right)+b\left(\Phi_{1} \wedge \Phi_{2}\right)$.

Now, since $\mathcal{R}_{i+1}\left(\Phi_{1} \wedge \Phi_{2}\right)$ is the disjoint union of

$$
\begin{gathered}
\mathcal{R}_{i+1}\left(\Phi \wedge \Psi \wedge\left(P_{i+1}=\delta_{i+1}\right)\right) \text { and } \mathcal{R}_{i+1}\left(\Phi \wedge \Psi \wedge\left(P_{i+1}=-\delta_{i+1}\right)\right), \\
b\left(\Phi_{1} \wedge \Phi_{2}\right)=b\left(\Phi \wedge \Psi \wedge\left(P_{i+1}=\delta_{i+1}\right)+b\left(\Phi \wedge \Psi \wedge\left(P_{i+1}=-\delta_{i+1}\right)\right) .\right.
\end{gathered}
$$

Moreover,

$$
\begin{gathered}
b\left(\Phi_{1}\right)=b\left(\Phi \wedge \Psi \wedge\left(P_{i+1} \geq 2 \delta_{i+1}\right)\right)+b\left(\Phi \wedge \Psi \wedge\left(P_{i+1} \leq-2 \delta_{i+1}\right)\right), \\
b\left(\Phi_{2}\right)=b\left(\Phi \wedge \Psi \wedge\left(P_{i+1}=0\right)\right) .
\end{gathered}
$$

Indeed, by Hardt's triviality theorem [5], denoting $F_{t}=\left\{x \in \mathcal{R}_{i}(\Phi \wedge \Psi) \mid\right.$ $\left.P_{i+1}(x)=t\right\}$, there exists $t_{0} \in \mathrm{R}\left\langle\delta_{1}, \ldots, \delta_{i}\right\rangle$ such that $F_{\left[-t_{0}, 0\right) \cup\left(0, t_{0}\right]}=\{x \in$ $\left.\mathcal{R}_{i}(\Phi \wedge \Psi) \mid t_{0}^{2} \geq P_{i+1}(x)>0\right\}$ and $\left(\left[-t_{0}, 0\right) \times F_{-t_{0}}\right) \cup\left(\left(0, t_{0}\right] \times F_{t_{0}}\right)$, are homeomorphic, and moreover the homeomorphism can be chosen such that it is the identity on the fibers $F_{-t_{0}}$ and $F_{t_{0}}$.

This clearly implies that $F_{\left[\delta, t_{0}\right]}=\left\{x \in \mathcal{R}_{i+1}(\Phi \wedge \Psi) \mid t_{0} \geq P_{i+1}(x) \geq \delta\right\}$ and $F_{\left[2 \delta, t_{0}\right]}=\left\{x \in \mathcal{R}_{i+1}(\Phi \wedge \Psi) \mid t_{0} \geq P_{i+1}(x) \geq 2 \delta\right\}$ are homeomorphic.

Hence, $b\left(\Phi_{1}\right)=b\left(\Phi \wedge \Psi \wedge\left(P_{i+1} \geq 2 \delta_{i+1}\right)\right)+b\left(\Phi \wedge \Psi \wedge\left(P_{i+1} \leq-2 \delta_{i+1}\right)\right)$.

Note that $F_{0}=\mathcal{R}_{i+1}\left(\Phi \wedge \Psi \wedge\left(P_{i+1}=0\right)\right)$ and $F_{[-\delta, \delta]}=\mathcal{R}_{i+1}\left(\Phi_{2}\right)$. Thus, it remains to prove that $b\left(F_{[-\delta, \delta]}\right)=b\left(F_{0}\right)$. By Hardt's triviality theorem [5], for every $0<u<1$ there is a fiber-preserving semi-algebraic homeomorphism $\phi_{u}$ from 
$F_{[-\delta,-u \delta]}$ to $[-\delta,-u \delta] \times F_{-u \delta}$ (resp. a semi-algebraic homeomorphism $\psi_{u}$ from $F_{[u \delta, \delta]}$ to $\left.[u \delta, \delta] \times F_{u \delta}\right)$. We define a continuous semi-algebraic homotopy $g$ from the identity of $F_{[-\delta, \delta]}$ to $\lim _{\delta_{i+1}}$ from $F_{[-\delta, \delta]}$ to $F_{0}$ as follows:

- $g(0,-)$ is $\lim _{\delta_{i+1}}$,

- for $0<u \leq 1, g(u,-)$ is the identity on $F_{[-u \delta, u \delta]}$ and sends $F_{[-\delta,-u \delta]}$ (resp. $\left.F_{[u \delta, \delta]}\right)$ to $F_{-u \delta}\left(\right.$ resp. $\left.F_{u \delta}\right)$ by $\phi_{u}$ (resp. $\psi_{u}$ )followed by the projection on $F_{u \delta}\left(\right.$ resp. $\left.F_{-u \delta}\right)$.

Thus $b\left(F_{[-\delta, \delta]}\right)=b\left(F_{0}\right)$. Finally, $b(\Phi \wedge \Psi) \leq \sum_{\psi \in \Sigma_{i+1}} b(\Phi \wedge \Psi \wedge \psi)$.

Proof of Proposition 3. Starting from the formula $\Phi$, apply Lemma 4.2 with $\Psi$ the empty formula. Now, repeatedly apply Lemma 4.2 to the terms appearing on the right-hand side of the inequality obtained, noting that for any $\Psi \in \Sigma_{\leq s}$,

- either $\mathcal{R}_{s}(\Phi \wedge \Psi)=\mathcal{R}_{s}(\Psi)$, and $\mathcal{R}_{s}(\Psi) \subset \mathcal{R}_{s}(\Phi)$,

- or $\mathcal{R}_{s}(\Phi \wedge \Psi)=\emptyset$.

Using an argument analogous to that used in the proof of Theorem 1.1 we prove the following proposition.

\section{Proposition 4.}

$$
\sum_{\Psi \in \Sigma_{\leq s}} b(\Psi) \leq \sum_{j=0}^{k^{\prime}-i}\left(\begin{array}{l}
s \\
j
\end{array}\right) 6^{j} d(2 d-1)^{k-1} .
$$

We first prove the following Lemma 4.3 and Lemma 4.4

Let $\mathcal{P}=\left\{P_{1}, \ldots, P_{j}\right\} \subset R\left[X_{1}, \ldots, X_{k}\right]$, and let

$$
Q_{i}=P_{i}^{2}\left(P_{i}^{2}-\delta_{i}^{2}\right)^{2}\left(P_{i}^{2}-4 \delta_{i}^{2}\right)
$$

Let $W_{0}$ (resp. $\left.W_{1}\right)$ be the union of the sets $\mathcal{R}\left(Q_{i}=0, \operatorname{Ext}\left(Z_{r}, \mathrm{R}\left\langle\delta_{1}, \ldots, \delta_{j}\right\rangle\right)\right)$ (resp. $\left.\mathcal{R}\left(Q_{i} \geq 0, \operatorname{Ext}\left(Z_{r}, \mathrm{R}\left\langle\delta_{1}, \ldots, \delta_{j}\right\rangle\right)\right)\right)$, with $1 \leq i \leq j$.

Notice that $W_{1}=\bigcup_{\Psi \in \Sigma_{\leq s}} \mathcal{R}(\Psi)$.

\section{Lemma 4.3.}

$$
b_{i}\left(W_{0}\right) \leq\left(6^{j}-1\right) d(2 d-1)^{k-1} .
$$

Proof. The set $\mathcal{R}\left(\left(P_{i}^{2}\left(P_{i}^{2}-\delta_{i}^{2}\right)^{2}\left(P_{i}^{2}-4 \delta_{i}^{2}\right)=0\right), Z_{r}\right)$ is the disjoint union of

$$
\begin{gathered}
\mathcal{R}\left(P_{i}=0, \operatorname{Ext}\left(Z_{r}, \mathrm{R}\left\langle\delta_{1}, \ldots, \delta_{j}\right\rangle\right)\right), \\
\mathcal{R}\left(P_{i}=\delta_{i}, \operatorname{Ext}\left(Z_{r}, \mathrm{R}\left\langle\delta_{1}, \ldots, \delta_{j}\right\rangle\right)\right), \\
\mathcal{R}\left(P_{i}=-\delta_{i}, \operatorname{Ext}\left(Z_{r}, \mathrm{R}\left\langle\delta_{1}, \ldots, \delta_{j}\right\rangle\right)\right), \\
\mathcal{R}\left(P_{i}=2 \delta_{i}, \operatorname{Ext}\left(Z_{r}, \mathrm{R}\left\langle\delta_{1}, \ldots, \delta_{j}\right\rangle\right)\right),
\end{gathered}
$$

and

$$
\mathcal{R}\left(P_{i}=-2 \delta_{i}, \operatorname{Ext}\left(Z_{r}, \mathrm{R}\left\langle\delta_{1}, \ldots, \delta_{j}\right\rangle\right)\right) .
$$

Moreover, the $i$-th Betti numbers of their union $W_{0}$ is bounded by the sum of the Betti numbers of all possible non-empty sets that can be obtained by taking intersections of these sets using inequality 3.1 of Proposition 2

The number of possible $\ell$-ary intersections is $\left(\begin{array}{l}j \\ \ell\end{array}\right)$. Each such intersection is a disjoint union of $5^{\ell}$ algebraic sets. The $i$-th Betti number of each of these algebraic sets is bounded by $d(2 d-1)^{k-1}$ by (2.1).

Thus, $b_{i}\left(W_{0}\right) \leq \sum_{\ell=1}^{j}\left(\begin{array}{l}j \\ \ell\end{array}\right) 5^{\ell} d(2 d-1)^{k-1}=\left(6^{j}-1\right) d(2 d-1)^{k-1}$. 


\section{Lemma 4.4.}

$$
b_{i}\left(W_{1}\right) \leq\left(6^{j}-1\right) d(2 d-1)^{k-1}+b_{i}\left(Z_{r}\right) .
$$

Proof. Let $F=\mathcal{R}\left(\bigwedge_{1 \leq i \leq j} Q_{i} \leq 0 \vee \bigvee_{1 \leq i \leq j} Q_{i}=0, \operatorname{Ext}\left(Z_{r}, \mathrm{R}\left\langle\delta_{1}, \ldots, \delta_{i}\right\rangle\right)\right)$. Now, $W_{1} \cup F=Z_{r}$ and $W_{1} \cap F=W_{0}$. Using inequality (2.2) and the fact that

$$
b_{i}\left(Z_{r}\right)=b_{i}\left(\operatorname{Ext}\left(Z_{r}, \mathrm{R}\left\langle\delta_{1}, \ldots, \delta_{i}\right\rangle\right)\right),
$$

we deduce that $b_{i}\left(W_{1}\right) \leq b_{i}\left(W_{1} \cap F\right)+b_{i}\left(W_{1} \cup F\right)=b_{i}\left(W_{0}\right)+b_{i}\left(Z_{r}\right)$. We conclude using Lemma 4.3 .

Proof of Proposition 4. Since for all $i<k^{\prime}, b_{i}\left(Z_{r}\right)+b_{k^{\prime}}\left(Z_{r}\right) \leq d(2 d-1)^{k-1}$ by (2.1), we have that

$$
\sum_{\Psi \in \Sigma_{\leq s}} b(\Psi)=b\left(W_{1}\right) \leq b_{k^{\prime}}\left(Z_{r}\right)+\sum_{j=1}^{k^{\prime}-i}\left(\begin{array}{l}
s \\
j
\end{array}\right)\left(6^{j} d(2 d-1)^{k-1}\right)
$$

using inequality 3.2 of Proposition 2 and Lemma 4.4. Thus,

$$
\sum_{\Psi \in \Sigma_{\leq s}} b(\Psi) \leq \sum_{j=0}^{k^{\prime}-i}\left(\begin{array}{l}
s \\
j
\end{array}\right) 6^{j} d(2 d-1)^{k-1} .
$$

Proof of Theorem 4.1. The statement follows from Proposition 4 and Proposition 3

\section{REFERENCES}

[1] S. Basu Different bounds on the Different Betti Numbers of Semi-algebraic Sets, Discrete and Computational Geometry, 30:65-85, 2003. MR1991587(2004f:14082)

[2] S. BAsu On Bounding the Betti Numbers and Computing the Euler Characteristics of Semi-algebraic Sets, Discrete and Computational Geometry, 22:1-18, 1999. MR1692627 (2000d:14061)

[3] S. Basu, R. Pollack, M.-F. Roy On the number of cells defined by a family of polynomials on a variety, Mathematika, Vol 43, 120-126, 1996. MR1401711 (97h:14076)

[4] S. Basu, R. Pollack, M.-F. Roy Algorithms in real algebraic geometry. Algorithms and Computation in Mathematics, Vol 10, Springer-Verlag (2003). MR,1998147

[5] J. Bochnak, M. Coste, M.-F. Roy Real algebraic geometry. Ergebnisse der Mathematik und ihrer Grenzgebiete. 3. Folge, Bd. 36, Berlin : Springer-Verlag (1998).

[6] R. M. Hardt Semi-algebraic Local Triviality in Semi-algebraic Mappings Amer. J. Math. 102, 291-302 (1980). MR0564475 (81d:32012)

[7] O. A. Oleinik, I. B. Petrovski On the topology of real algebraic surfaces, Izv. Akad. Nauk SSSR 13, 389-402, (1949). MR0034600 (11:613h)

[8] J. Milnor On the Betti numbers of real varieties, Proc. AMS 15, 275-280, (1964). MR.0161339(28:4547)

[9] E. H. Spanier Algebraic Topology, McGraw-Hill Book Company, 1966. MR0210112 (35:1007)

[10] R. Тном Sur l'homologie des variétés algébriques réelles, Differential and Combinatorial Topology, Ed. S.S. Cairns, Princeton Univ. Press, 255-265, (1965). MR0200942 (34:828)

School of Mathematics, Georgia Institute of Technology, Atlanta, Georgia 30332 E-mail address: saugata@math.gatech.edu

Courant Institute of Mathematical Sciences, New York University, New York, New YORK 10012

E-mail address: pollack@cims.nyu.edu

iRmaR (URA CNRS 305), Université de Rennes, Campus de Beaulieu 35042 Rennes CEDEX, France

E-mail address: mfroy@maths.univ-rennes1.fr 